\title{
Underground Nuclear Astrophysics: pushing direct mea- surements toward the Gamow window
}

\author{
Paolo Prati ${ }^{1, *}$ \\ ${ }^{1}$ Università di Genova and INFN Genova, Italy
}

\begin{abstract}
The aim of experimental nuclear astrophysics is to provide information on the nuclear processes involved in astrophysical scenarios at the relevant energy range. However, the measurement of the cross section of nuclear reactions at low energies present formidable difficulties due to the very low reaction rates often overwhelmed by the background. Several approaches have been proposed and exploited to overcome such severe obstacles: in such frame, the idea to install a low energy - high intensity ion accelerator deep underground, to gain high luminosity while reducing the cosmic ray background, brought more than 25 years ago, to the pilot LUNA experiment. LUNA stands for Laboratory for Underground Nuclear Astrophysics: in the cave under the Gran Sasso mountain (in Italy) first a $50 \mathrm{kV}$ and then a $400 \mathrm{kV}$ single-ended accelerator for protons and alphas were deployed and produced plenty of data mainly on reactions of the H-burning phase in stars. Recently, similar facilities have been installed and/or proposed in other underground laboratories in US and China. LUNA as well is going to make a big step forward, with a new machine in the MV range which will be able to provide intense beams of protons, alphas and carbon ions. The rationale of underground nuclear astrophysics will be presented together with the last updates on the ongoing research programs.
\end{abstract}

\section{Introduction}

Stellar evolution and related nucleosynthesis are the key to understand the origin of the chemical elements and many astrophysical problems Many nuclear reactions, either strong or weak processes, are of astrophysical interest. For most of them, the knowledge of their cross sections (or reaction rates) at relatively low energy is required to understand the synthesis of the elements. These reactions can even influence the physical parameters of stellar interiors, such as temperature and density, and, in turn, determine the stellar lifetimes. Nuclear reactions that generate energy and synthesize elements take place inside the stars in a relatively narrow energy window: the Gamow peak. The extremely low value of the cross-section inside the Gamow peak has always prevented its measurement in a laboratory at the Earth's surface, where the signal to background ratio is usually too small because of cosmic ray interactions. In order to explore this new domain of nuclear astrophysics LUNA (Laboratory for Underground Nuclear Astrophysics) started in 1991 its activity by installing a $50 \mathrm{kV}$ electrostatic accelerator underground at Gran Sasso (Italy), followed in the year 2000 by a second 400 $\mathrm{kV}$ single-ended machine [1]. The qualifying features of both accelerators are a very small

\footnotetext{
*e-mail: paolo.prati@ge.infn.it
} 
beam energy spread and a very high beam current even at low energy. The extremely low laboratory background allowed to perform experiments with very small count rates, down to a few events per month. This way, the important reactions responsible for the hydrogen burning in the Sun could be studied down to the relevant stellar energies $[2,3]$. In the past LUNA could exclude the ghost resonance in the cross section of ${ }^{3} \mathrm{He}\left({ }^{3} \mathrm{He}, 2 \mathrm{p}\right)^{4} \mathrm{He}$ within the solar Gamow peak and provided the lowest-energy, precise measurement of ${ }^{3} \mathrm{He}\left({ }^{4} \mathrm{He}, \gamma\right){ }^{7} \mathrm{Be}$ which has firmly established the correctness of the nuclear ingredients of the proton-proton chain in the standard solar model. Furthermore, the measurement of the bottle-neck reaction of the CNO cycle, ${ }^{14} \mathrm{~N}(\mathrm{p}, \gamma){ }^{15} \mathrm{O}$, at very low energy provided a cross section lower by about a factor of two than existing extrapolations, decreasing by the same amount the flux of CNO neutrinos from the Sun.

Actually, LUNA results made possible the study of the metallicity of the Sun interior through the measurement of the CNO solar neutrinos. Several years ago, at the end of the solar phase, a rich program started devoted to the study of Big Bang Nucleosynthesis [4] and of the nucleosynthesis of the elements through the $\mathrm{CNO}, \mathrm{Ne}-\mathrm{Na}$ and $\mathrm{Mg}-\mathrm{Al}$ [5] cycles.

The current $400 \mathrm{kV}$ LUNA accelerator and the unique low-background conditions of the underground LNGS laboratory have been and still are the perfect combination for the study of most of the proton-capture reactions involved in the stellar $\mathrm{H}$ burning. Such decisive achievements have motivated the proposals for two similar facilities currently under construction in the Republic of China and in the United States. LUNA as well is going to be upgraded by the deployment at Gran Sasso of a new 3.5 MV accelerator for protons, alphas and carbon ions with beam intensities overcoming $1 \mathrm{~mA}$ (for protons, about one half for alphas and 1 tenth for carbon).

\section{Underground nuclear astrophysics: the LUNA facility}

The $400 \mathrm{kV}$ accelerator (LUNA400), presently in operation, is an electrostatic accelerator produced by High Voltage Engineering Europe (Figure 1). The High Voltage is provided by a stabilized Inline-Cockcroft-Walton power supply. The long-term energy stability has been measured to remain within $2 \mathrm{eV}[6]$. A radio frequency ion source is mounted directly on the accelerator tube and the ions are extracted by a voltage applied to an electrode inside the tube itself. The source can deliver, continuolsy for two months, up to $1 \mathrm{~mA}$ intensity for hydrogen and up to $0.5 \mathrm{~mA}$ for helium. Thus, the accelerator tank has to be opened periodically to change the ion source. The beam energy was calibrated through the non-resonant radiative capture reaction ${ }^{12} \mathrm{C}(\mathrm{p}, \gamma){ }^{13} \mathrm{~N}[6]$ and exploiting the resonances of $(\mathrm{p}, \gamma)$ captures on ${ }^{23} \mathrm{Na}$, ${ }^{25} \mathrm{Mg}$ and ${ }^{26} \mathrm{Mg}$.

At LUNA, the ions can be sent into one of two beam lines with two different target setups, i.e. solid and gas target. The solid target station is mounted at the end of a copper pipe cooled by liquid nitrogen: this acts as cold trap to prevent carbon buildup. The target holder also serves as a Faraday cup to measure the beam intensity. The other beam line is instead equipped with a windowless gas target [7] which is placed downstream a magnet to bend the beam to $45^{\circ}$, a vertical steering magnet, and several vacuum pumps, necessary to lower the pressure to about $10^{-7}$ mbar inside the accelerator. A set of apertures with decreasing diameter connect the accelerator to the reaction chamber. The current lost at the target entrance (a collimator $40 \mathrm{~mm}$ long and with a diameter of $7 \mathrm{~mm}$ ) is lower than $1 \%$. The beam stop is made by a copper block wich acts as calorimeter to measure the beam intensity with a typical accuracy lower than $2 \%$. 


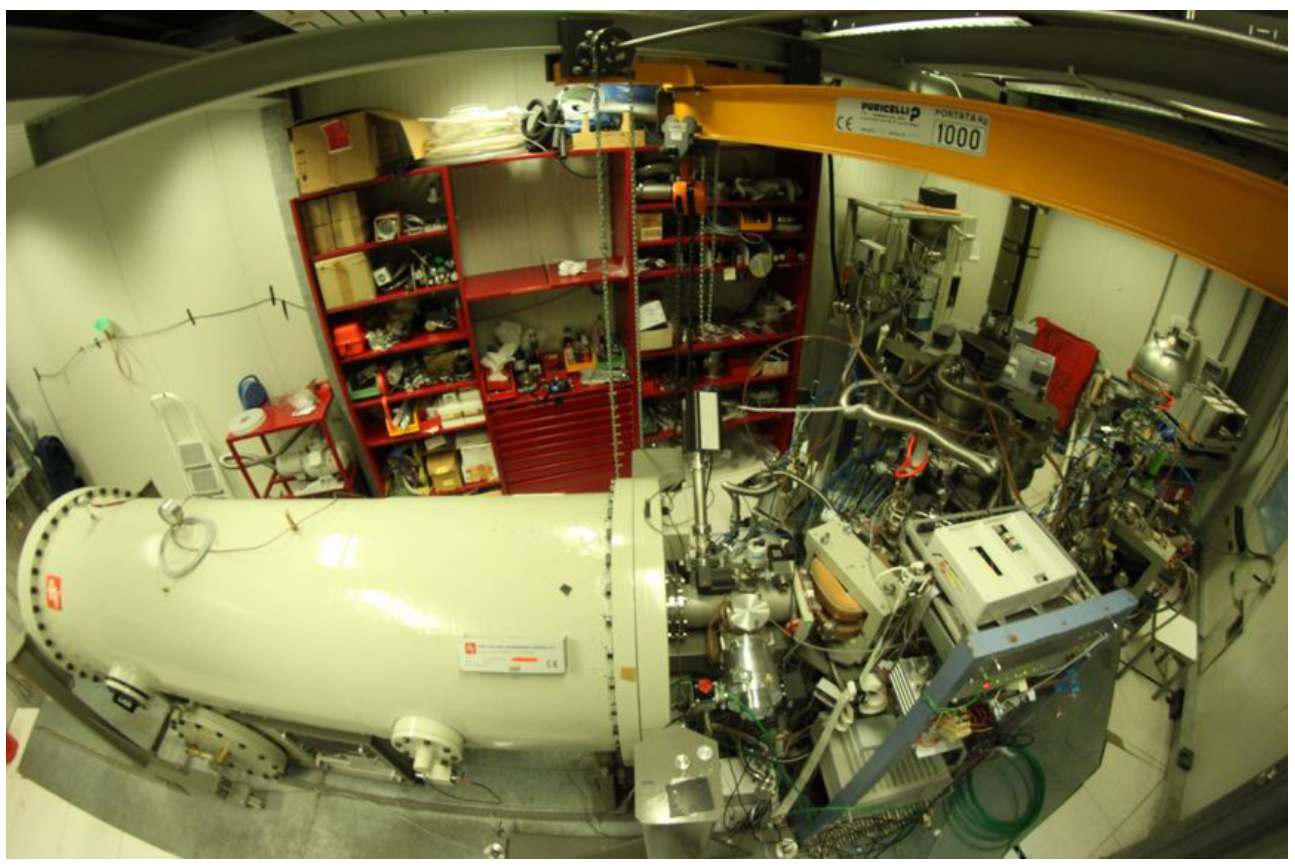

Figure 1. The LUNA400 facility in operation in the underground laboratory of Gran Sasso, Italy

\section{The LUNA-MV project}

The LUNA MV project has been developed to study the phases of stellar evolution successive to the Hydrogen quiescient burning, in particular the helium and carbon burning phases. To face the challenge, a new 3.5 MV single-ended accelerator will be installed in Gran Sasso in the forthcoming months. The new accelerator will deliver hydrogen, helium and carbon high-intensity beams. The direct measurement of the cross section of the ${ }^{12} \mathrm{C}(\alpha, \gamma){ }^{16} \mathrm{O}$ and ${ }^{12} \mathrm{C}+{ }^{12} \mathrm{C}$ reactions at the relevant astrophysical energies are the main goals of the project. The sources of neutrons for stellar nucleosynthesis beyond iron, are another open problem to be faced at the new LUNA-MV facility.

In particular, ${ }^{13} \mathrm{C}(\alpha, n){ }^{16} \mathrm{O}$ and ${ }^{22} \mathrm{Ne}(\alpha, n){ }^{25} \mathrm{Mg}$ will be both part of the scientific program for the first years of activity and the experiments with LUNA MV will allow the direct measurement of their cross sections in the energy range of astrophysical interest.

The new accelerator facility will be installed inside the Hall B of the Gran Sasso underground laboratory. The accelerator will be deployed inside a new room with concrete walls (Figure 2). The concrete walls and the room roof (thickness of $80 \mathrm{~cm}$ ) serve as neutron shielding to prevent any possible undesidered background to the other experiments installed in the same site. The shileding guarantees that, in the worst operating conditions (i.e.: maximum energy and beam intensity), the neutron flux just outside the accelerator room will be about one tenth of the laboratory natural background (mainly due to the rocks internal radiaoctivity).

The LUNA-MV accelerator (Figure 3) is a custom Inline Cockcroft Walton accelerator, manufactured at High Voltage Engineering Europe (HVEE) [8]. The Terminal Voltage (TV) will range from 0.2 to 3.5 MV. As with the present LUNA400 accelerator, ion beams of $\mathrm{H}+$, ${ }^{4} \mathrm{He}+,{ }^{12} \mathrm{C}+$ and ${ }^{12} \mathrm{C}++$ in the energy range from 0.350 to $7 \mathrm{MeV}$ could be sent to two different 


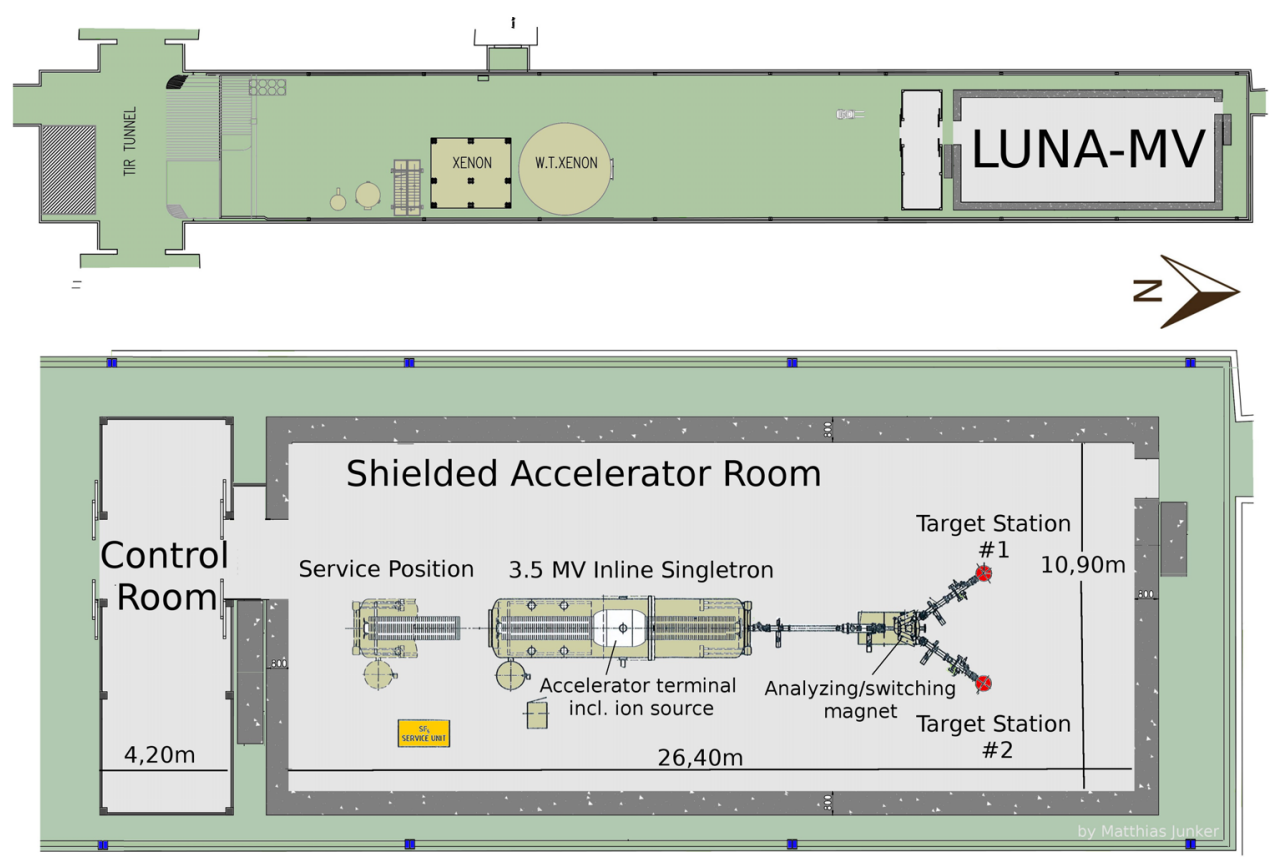

Figure 2. Layout of the new LUNA-MV accelerator facility in the Hall B of the underground laboratory of Gran Sasso, Italy

beam lines (see Figure 3). Beam intensity is widely tunable with maximum values of about $1 \mathrm{~mA}$ for protons, $0.5 \mathrm{~mA}$ for alphas and $0.15 \mathrm{~mA}$ for Carbon ions. Energy spread and long term stability, both essential for an accurate determination of the cross sections under study, will be of the same level of the previous LUNA400 accelerator. While a scientific progrma for the first years of life of the new accelerator has been submitted and approved by the Gran Sasso Scientific Committee, the new facility will be open to the international community. A PAC (Program Advisory Committee) will be established to select proposal and assign the proper beam time.

\section{References}

[1] H. Costantini, A. Formicola, G. Imbriani, M. Junker, C. Rolfs, F. Strieder, Rep. Prog. Phys. 72, 086301 (2009)

[2] C. Broggini, D. Bemmerer, A. Caciolli, D. Trezzi, Progress in Particle and Nuclear Physics 98, 55 (2018)

[3] F. Cavanna, P. Prati, International Journal of Modern Physics A 33, 1843010 (2018)

[4] D. Trezzi, M. Anders, M. Aliotta, A. Bellini, D. Bemmerer, A. Boeltzig, C. Broggini, C. Bruno, A. Caciolli, F. Cavanna et al., Astroparticle Physics 89, 57 (2017)

[5] A. Boeltzig, C.G. Bruno, F. Cavanna, S. Cristallo, T. Davinson, R. Depalo, R.J. deBoer, A. Di Leva, F. Ferraro, G. Imbriani et al., The European Physical Journal A 52, 75 (2016)

[6] A. Formicola, G. Imbriani, M. Junker, D. Bemmerer, R. Bonetti, C. Broggini, C. Casella, P. Corvisiero, H. Costantini, G. Gervino et al., Nucl. Inst. Meth. A 507, 609 (2003)

[7] C. Casella, H. Costantini, A. Lemut, B. Limata, D. Bemmerer, R. Bonetti, C. Broggini, L. Campajola, P. Cocconi, P. Corvisiero et al., Nucl. Inst. Meth. A 489, 160 (2002) 

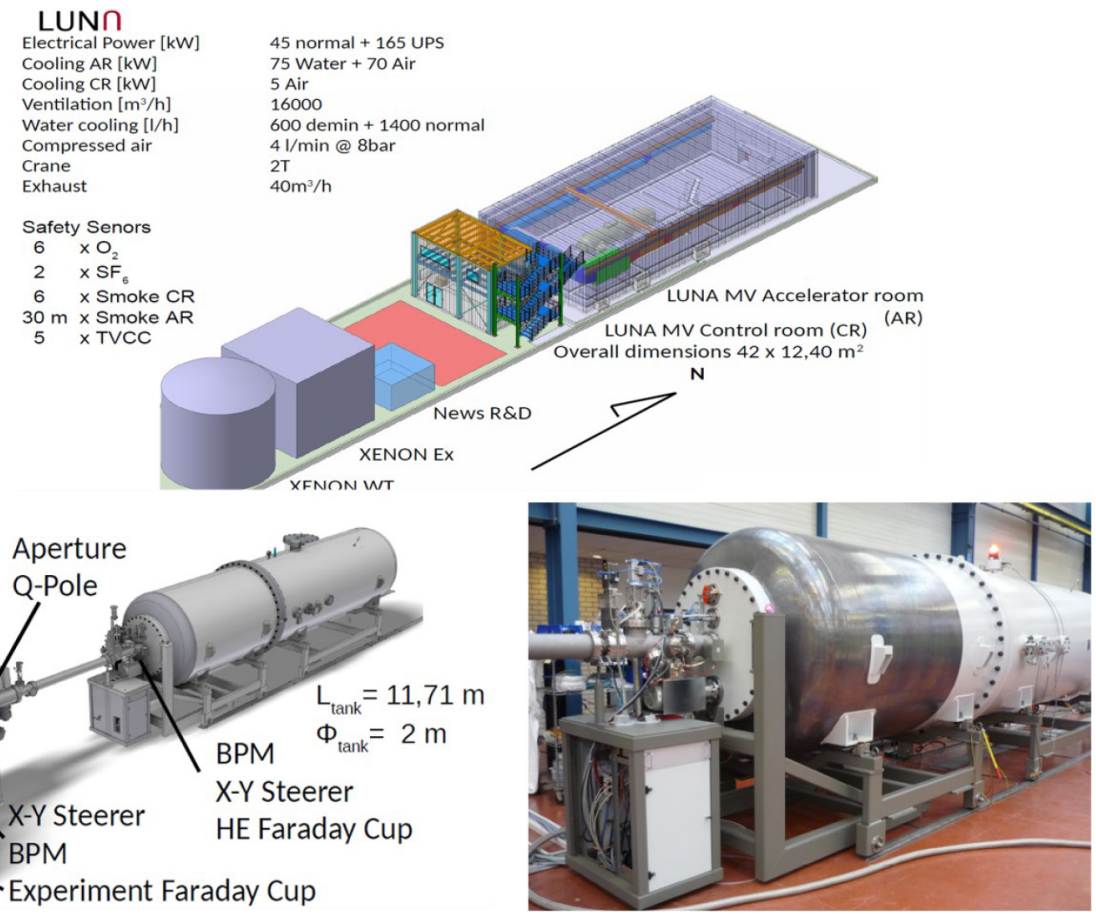

Figure 3. Main features of the LUNA-MV accelerator and of related plant. A picture of the accelerator taken during the assembly in the HVEE factory is also shown

[8] A. Sen, G. Dominnguez-Canizares, N. Podaru, D. Mous, M. Junker, G. Imbriani, V. Rigato, Nuclear Instruments and Methods in Physics Research Section B: Beam Interactions with Materials and Atoms 450, 390 (2019), the 23rd International Conference on Ion Beam Analysis 